\title{
Knee joint reconstruction using regional flaps: an aesthetic approach to extensive defects following fourth-degree contact burns
}

\author{
Suk Joon Oh, Jong Cheol Kang \\ Department of Burn Reconstructive \\ Surgery, Bestian Seoul Hospital, Seoul, \\ Korea
}

\begin{abstract}
Flap surgery may be required in cases of severe contact burns. The authors report a patient with an exposed knee joint. A 47-year-old man experienced a wide and deep contact burn on his right knee from a stove while under the influence of alcohol. The patient underwent escharectomy, medial and lateral gastrocnemius muscle flap surgery, pedicled anterolateral thigh flap surgery with a meshed scalp split-thickness skin graft, and secondary antegrade peroneal fasciocutaneous flap surgery with a thigh splitthickness skin graft. The patient was able to walk with a hinged brace at 2 months after surgery.
\end{abstract}

Keywords Knee joint / Burns / Surgical flaps / Transplants

\section{INTRODUCTION}

The reconstruction of complex and extensive defects involving the knee joint represents an aesthetic and functional challenge. The knee joint is one of the most complex structures of the body in terms of dimensional architecture, thickness, reliability, and its functional role. Defects affecting the total thickness of the knee joint have negative effects on patients' social function, quality of life, physical health, and satisfaction with their appearance when wearing shorts. Therefore, aesthetic outcomes after the coverage of soft tissue defects for reconstruction are important.

Soft tissue coverage for defects around the knee joint has been performed using different types of pedicled flaps and free flaps [1].

Gastrocnemius muscle flaps have been used for decades to reconstruct defects of the proximal tibia and knee caused by trauma, tumors, and infections around the knee, and to reconstruct extensor mechanism discontinuities with and without total joint arthroplasty [2]. Furthermore, antegrade peroneal fasciocutaneous flaps

Received: Nov 27, 2020 Revised: Dec 10, 2020 Accepted: Dec 15, 2020 Correspondence: Suk Joon Oh Department of Burn Reconstructive Surgery, Bestian Seoul Hospital, 429 Dogok-ro, Gangnam-gu, Seoul 06208, Korea Tel: +82-70-7609-9321, Fax: +82-70-7005-4233, E-mail: sjoh46@nate.com

Copyright @ 2021 The Korean Society for Aesthetic Plastic Surgery.

This is an Open Access article distributed under the terms of the Creative Commons Attribution Non-Commercial License (https://creativecommons.org/licenses/by-nc/4.0/) which permits unrestricted non-commercial use, distribution, and reproduction in any medium, provided the original work is properly cited. www.e-aaps.org may be useful for reconstruction of the inferior knee joint.

Herein, the authors report a case of a fourth-degree contact burn on the knee joint that was reconstructed using medial and lateral gastrocnemius muscle flaps, pedicled anterolateral thigh flap, peroneal fasciocutaneous flap, and split-thickness skin grafts (SSGs) harvested from the scalp and thigh. Four types of pedicled flaps were needed because this patient had an extensive, deep defect in the right knee joint, which is an exceedingly rare condition.

\section{CASE REPORT}

A 47-year-old man suffered from a contact burn from a stove (total body surface area, $4 \% ; 200 \mathrm{~cm}^{2}$ ) on the anterolateral aspect of his right knee while under the influence of alcohol (Fig. 1A). The distal part of the vastus lateralis muscle, the lateral part of the quadriceps tendon, the lateral retinaculum, and the anterior cortex of the patella were damaged, and the iliotibial band was exposed after escharectomy. Preoperative magnetic resonance imaging (MRI) showed damage to the vastus lateralis muscle without bone involvement.

Removal of the damaged tissue and cortex of the patella resulted in a wide wound, exposing the knee joint (Fig. 1B). Medial and lateral gastrocnemius muscle flaps were harvested through a posterior midline incision in the lower leg (Fig. 1C). The vastus lateralis muscle defect, the exposed quadriceps tendon, and the iliotibial band were covered with the lateral gastrocnemius muscle flap, and the decorticated patella and patellar tendon were covered with the medial gastrocnemius muscle flap. An anterolateral thigh flap and 

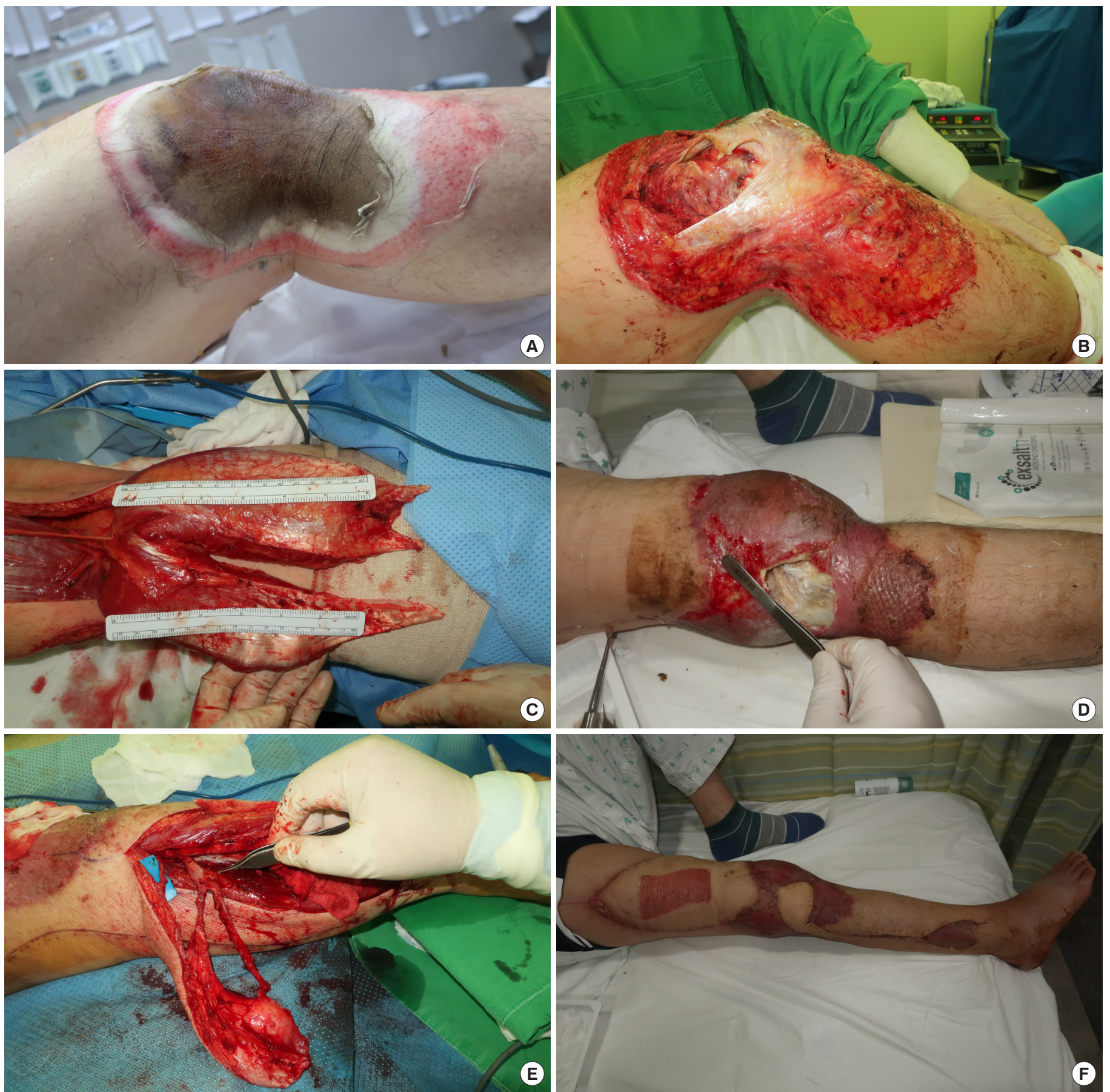

Fig. 1. Reconstructive procedures of the right knee joint. (A) A 47-year-old man developed a contact burn on the anterolateral aspect of his right knee from a stove while under the influence of alcohol. (B) Removal of damaged tissue of the distal part of the vastus lateralis muscle, the lateral part of the quadriceps tendon, the lateral retinaculum, and the cortex of the patella caused a wide wound, resulting in knee joint exposure. (C) Medial and lateral gastrocnemius muscle flaps were harvested through the midline incision of the lower leg. (D) The knee joint was exposed due to loss of the skin graft over the iliotibial band. (E) The peroneal fasciocutaneous flap included three perforators. (F) The wide defect of the right knee was reconstructed by four flaps, including the scalp and thigh split-thickness skin grafts.

meshed scalp SSG (0.406-mm thick, $250 \mathrm{~cm}^{2}$ in area) covered the medial and lateral gastrocnemius muscle flaps and the remaining wound on the lower leg. The knee joint was exposed due to loss of the skin graft over the iliotibial band (Fig. 1D). Therefore, a second antegrade peroneal fasciocutaneous flap was needed.

This peroneal fasciocutaneous flap included three perforators 

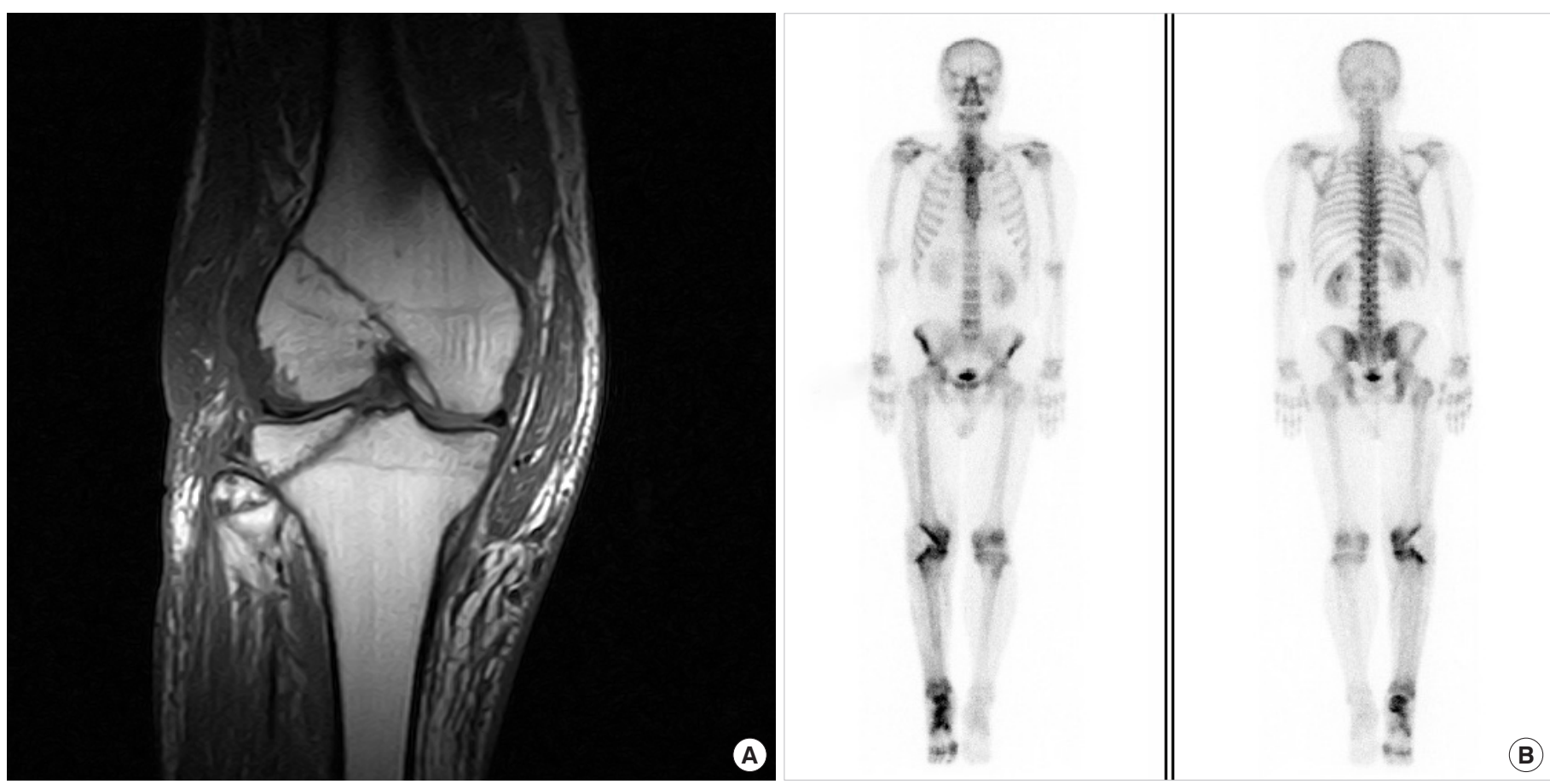

Fig. 2. Postoperative magnetic resonance imaging (MRI) and bone scan. (A) The MRI findings showed fracture-like lines on the left condyle of the femur and tibia and the head of the fibula at 2 months after surgery. (B) The bone scans showed a cold defect on the lateral side of the right knee and increased bone uptake along the fracture lines of the right knee 2 months after surgery.

(Fig. 1E). The defect on the right knee joint was reconstructed again with an antegrade peroneal fasciocutaneous flap and thigh SSG (0.305-mm thick, $40 \mathrm{~cm}^{2}$ in area). The wide defect of the right knee was reconstructed using four flaps, including the scalp and thigh SSGs (Fig. 1F). The patient was satisfied with the aesthetic outcomes following knee joint reconstruction using multiple pedicled flaps. Therefore, these reconstruction methods may result in higher satisfaction with the knee joint shape and graft insetting among patients with extensive defects of the knee joint, and may achieve aesthetically satisfactory results with safe tailoring. The patent was able to walk with a hinged brace 2 months after surgery.

Preoperative MRI did not show bony involvement. However, postoperative MRI (Fig. 2A) and bone scans of the right knee using Tc-99m-HDP (25 mCi) (Fig. 2B) showed partial avascular ischemic damage of the lateral condyles of the femur and tibia and the head of the fibula at 2 months after surgery.

\section{DISCUSSION}

Soft tissue defects may occur around the knee joint from wound breakdown following surgery or trauma and deep burns. Early aseptic closure of the exposed knee joint should be performed to achieve adequate functional and cosmetic results with minimal donor site morbidities. Various reconstructive methods exist, and the appropriate method is selected depending on the location, size, and depth of the defect relative to the knee joint $[1,3]$.

A free musculocutaneous flap could have been an option for reconstruction in this patient, but our clinic was unable to perform microsurgery. Therefore, the authors chose regional flaps.

The medial head of the gastrocnemius muscle in adults measures $15-20 \mathrm{~cm}$ in length and $8 \mathrm{~cm}$ in width. The lateral head of the gastrocnemius muscle measures $12-17 \mathrm{~cm}$ in length and $6 \mathrm{~cm}$ in width [4]. The gastrocnemius muscle flap is considered to be the main pedicled flap for soft-tissue defect reconstructions in the knee. When this flap is not available, pedicled options include a reversed anterolateral thigh flap or peroneal artery flap [5]. Little data have been published on the use of this peroneal flap for knee reconstruction $[6,7]$.

The extent of knee joint exposure in this present patient was large, and the defect was not properly reconstructed with medial and lateral gastrocnemius flaps and an anterolateral thigh flap. Therefore, a second antegrade peroneal fasciocutaneous flap was needed.

The wide fascia $(9 \times 10 \mathrm{~cm})$ of the antegrade peroneal fasciocutaneous flap reinforced the iliotibial band defect. The donor defect $\left(40 \mathrm{~cm}^{2}\right)$ was covered with a thigh SSG. The patient was able to walk with a hinged brace.

Avascular ischemic injuries to the lateral condyles of the femur and tibia, as well as to the fibular head of the right knee, gradually lead to bone destruction. Therefore, serial MRI studies of the right knee joint are needed during the postoperative period. If unstable 
movement occurs due to bony involvement in the right knee joint, knee joint replacement surgery may be required later.

Based on our experience, knee joint reconstruction using multiple pedicled flaps, including medial and lateral gastrocnemius muscle flaps, an anterolateral thigh flap, and peroneal flaps may be a useful way to obtain satisfactory functional and aesthetic outcomes.

\section{NOTES}

\section{Conflict of interest}

No potential conflict of interest relevant to this article was reported.

\section{Ethical approval}

The study was approved by the Institutional Review Board of Bestian Seoul Hospital (IRB No. 2020-10-001) and performed in accordance with the principles of the Declaration of Helsinki.

\section{Patient consent}

The patient provided written informed consent for the publication and the use of his images.

\section{ORCID}

Suk Joon Oh

https://orcid.org/0000-0001-7793-6198

Jong Cheol Kang

\section{REFERENCES}

1. R RB, Ramkumar S, Venkatramani H. Soft tissue coverage for defects around the knee joint. Indian J Plast Surg 2019;52:125-33.

2. Walton Z, Armstrong M, Traven S, et al. Pedicled rotational medial and lateral gastrocnemius flaps: surgical technique. J Am Acad Orthop Surg 2017;25:744-51.

3. Balakrishnan C, Flanagan TL, Klein JD, et al. Soft tissue coverage of the knee joint following burns. Can J Plast Surg 2006;14:163-6.

4. Saaiq M, Zimri FU. Clinical applications and outcome of proximally based medial gastrocnemius muscle flap. World J Plast Surg 2020;9:22-8.

5. Alshomer F, Alhujayri A, Althubaiti G. Pedicled peroneal artery perforator flap for knee defect reconstruction: case presentation and literature discussion. Plast Reconstr Surg Glob Open 2018;6:e2034.

6. Ruan HJ, Cai PH, Schleich AR, et al. The extended peroneal artery perforator flap for lower extremity reconstruction. Ann Plast Surg 2010;64:451-7.

7. Lu TC, Lin CH, Lin CH, et al. Versatility of the pedicled peroneal artery perforator flaps for soft-tissue coverage of the lower leg and foot defects. J Plast Reconstr Aesthet Surg 2011;64:386-93. 